\title{
Maxwell Homogenization Scheme in Micromechanics: an Overview
}

\author{
Igor Sevostianov ${ }^{1}$ \\ ${ }^{1}$ Department of Mechanical and Aerospace Engineering, New Mexico State University, Las Cruces, NM 88003, USA France
}

\begin{abstract}
The paper discuss the classical Maxwell's (1873) homogenization method and the problems appearing in its practical implications. The method equates the far fields produced by a set of inhomogeneities and by a fictitious domain with unknown effective properties. It is re-written in terms of the property contribution tensors. The shape of the effective inclusion substantially affects the overall elastic properties. The choice of this shape in the case of anisotropic composite is a non-trivial problem. Another problem is related to the possible appearance of artificial singularities in the process of homogenization. We illustrate the method by several examples.
\end{abstract}

\section{Introduction}

The Maxwell scheme appears to be one of the best homogenization techniques in terms of its applicability to cases of anisotropic multiphase composites and accuracy. In his original work [1], Maxwell considered the effective conductivity of a matrix of conductivity $k_{0}$ containing spherical inhomogeneities of conductivity $k_{1}$ and volume fraction $\varphi$. He considered a sufficiently large spherical domain possessing the yet unknown effective conductivity $k_{\text {eff }}$ embedded in the background material. He calculated the far-field asymptotics of the perturbation, by the inhomogeneities, of the externally applied electric field, in two different ways: (1) as a sum of far-fields generated by the small spheres treated as non-interacting ones, and (2) as the far-field generated by the mentioned large sphere (Fig. 1). Equating the two yields the effective conductivity in the form

$$
k_{\text {eff }}=k_{0} \frac{1+2 \varphi \Psi}{1-\varphi \Psi}
$$

where $\Psi \equiv\left(k_{1}-k_{0}\right) /\left(k_{1}+2 k_{0}\right)$. This relation is nonlinear in concentration of inhomogeneities. Comparing it with results for a periodic arrangement, Lord Rayleigh [2] found that Maxwell's formula remains sufficiently accurate up to volume fractions as high as 0.4. As discussed in [3], the formula (1) has been misinterpreted by several later authors who linearized it with respect to $\varphi$ - the operation that substantially worsened its accuracy $[4,5]$ :

$$
k_{\text {eff }}=k_{0}(1+3 \Psi \varphi)
$$

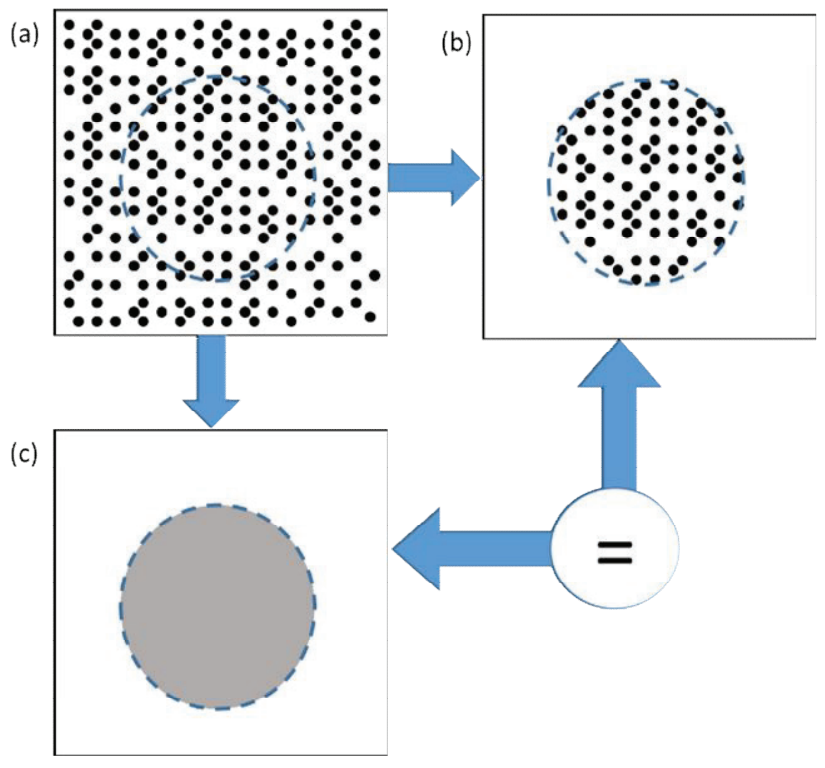

Fig. 1. Scheme of Maxwell's homogenization method. Effective properties of a composite (a) are calculated by equating effects produced by set of inhomogeneities embedded in the matrix material (b) and by fictitious domain having yet unknown effective properties (c).

This formula is sometimes erroneously identified with the original Maxwell's method (see, for example, [6]). Moreover, in contrast with the original Maxwell's result, formula (2) violates Hashin-Shtrikman bounds for the effective conductivity [3]. As further remark on the linearized formula (5.8.2), we note that Jeffrey [4], seeking to improve its accuracy, considered series expansion of $k_{\text {eff }}$ in powers of $\varphi$ and calculated the coefficient at the $\varphi^{2}$-term:

\footnotetext{
*Corresponding author: igor@nmsu.edu
} 


$$
\begin{gathered}
k_{\text {eff }} / k_{0}=(1+3 \Psi \varphi)+ \\
+\varphi^{2}\left(3 \Psi^{2}+\frac{3 \Psi^{3}}{4}+\frac{9 \Psi^{3}}{16} \frac{\alpha+2}{2 \alpha+3}+\frac{3 \Psi^{4}}{64}+\ldots\right)+o\left(\varphi^{2}\right)
\end{gathered}
$$

where $\alpha=k_{1} / k_{0}$. Fig. 2 compares Maxwell's original result (1) with the linearized version (2) and experimental data presented in [7] for (thermal) conductivity of epoxy containing particles of (a) silica ( $\alpha=k_{1} / k_{0}=7.69$ ), (b) silica coated aluminum nitride (SCAN) $(\alpha=1128)$, and (c) alumina $(\alpha=185)$. The insets show shapes of the ceramic particles. The plots also show the result (3) (the coefficients at $\varphi^{2}$-term were taken from the plot in Jeffrey's paper as 1.89, 4.51, and 4.35 for silica, SCAN and alumina particles respectively) and, also, the Hashin-Shtrikman bounds for conductivity. Note that adding the quadratic term to the linearized version moves it closer to the original (nonlinearized) Maxwell's formula but still violates the bounds.
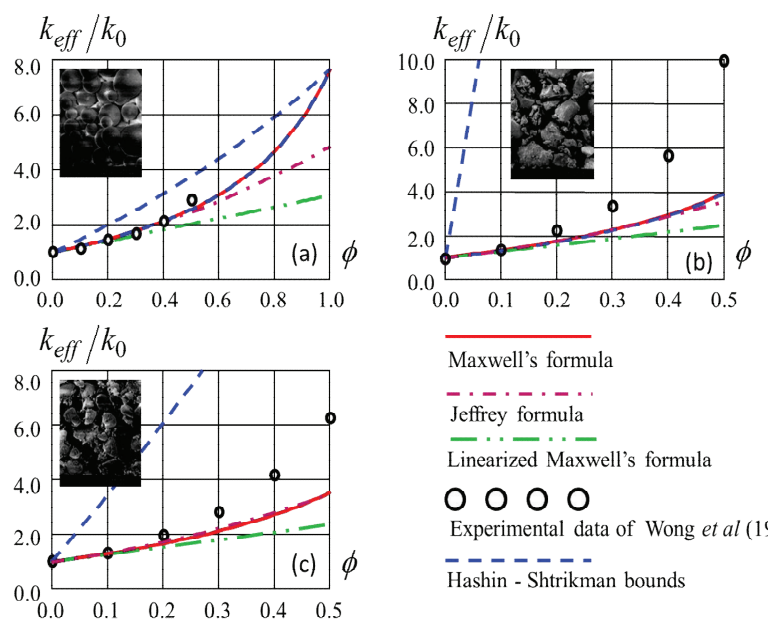

Linearized Maxwell's formula 0000 Experimental data of Wong et al (1999) $------$

Hashin - Shtrikman bounds

Fig. 2. Effective thermal conductivity of epoxy matrix filled with (a) silica, (b) SCAN (silica coated aluminum nitride) and (c) alumina particles: predictions by Maxwell's formula (1), its linearized variant (2), Jeffrey formula (3) are compared with experimental data of Wong and Bollampally (1999) and with Hashin-Shtrikman bounds for thermal conductivity. Insets show shape of particles of each material used for reinforcement.

Figure 2 also illustrates the importance of appropriate modeling of shapes of inhomogeneities. Indeed, the Maxwell's result for a composite with spherical silica particles coincides with experimental data up to the volume fraction of inhomogeneities 0.5. For SCAN and alumina particles having rather irregular shapes, modeling them as spherical ones leads to acceptable agreement with data at the volume fraction up to only 0.2 .

Recently, Maxwell's scheme started to attract increasing attention. We mention the works $[8,9]$, where the scheme was formulated for the elastic properties of a material containing either random or aligned ellipsoidal inhomogeneities of identical aspect ratios. In [10], Maxwell' scheme was applied to finding the elastic, electric and poroelastic properties of composite containing identical parallel ellipsoidal inhomogeneities. Following [11], we apply Maxwell's approach to effective elastic properties of composites containing nonspherical particles of generally diverse shapes and of arbitrary orientation distribution. To this end, we reformulate Maxwell's assumptions using property contribution tensors. These tensors - that, as discussed throughout the present book, are fundamental quantities in the problem of effective properties - are particularly useful for the Maxwell's scheme. Indeed, the far fields entering the scheme are directly expressed in terms of these tensors (Section 3.2): the far-field asymptotics of the elastic field generated by an inhomogeneity determines its contribution to the effective elastic properties and vice versa.

\section{Maxwell scheme in terms of property contribution tensors}

According to Maxwell's idea, we evaluate far-field perturbations due to inhomogeneities in two different ways and equate the results. Firstly, we evaluate this field as the one generated by a homogenized region $\Omega$ possessing the (yet unknown) effective properties. As discussed in [12], this field can be expressed in terms of the compliance contribution tensor $\boldsymbol{H}^{\text {eff }}$ of the domain $\Omega$ possessing the unknown effective properties. Secondly, we consider the sum of far fields generated by all the individual inhomogeneities within $\Omega$ (treated as non-interacting ones); these fields are expressed in terms of $\boldsymbol{H}$-tensors of the said inhomogeneities. Equating the two quantities yields:.

$$
\frac{V_{\Omega}}{V} \boldsymbol{H}^{e f f}=\frac{1}{V} \sum_{i} V_{i} \boldsymbol{H}^{i}
$$

The right hand side of the equation is known, however, the left hand side, besides being dependent on the effective properties, depends also on the shape of $\Omega$. Explicit analytic expressions for $\boldsymbol{H}^{\text {eff }}$ are available for the ellipsoidal shapes only [13], and in this case equation (4) can be explicitly solved for the effective properties. In the following, we consider domains $\Omega$ of the ellipsoidal shape, assuming that it provides sufficient flexibility. Then equation (4) can be specified as follows [13]:

$$
\frac{V}{V_{\Omega}}\left[\left(\boldsymbol{S}^{e f f}-\boldsymbol{S}^{0}\right)^{-1}+\boldsymbol{Q}_{\Omega}\right]=V\left[\sum_{i} V_{i} \boldsymbol{H}^{(i)}\right]^{-1}
$$

where $\boldsymbol{Q}_{\Omega}$ is fourth-rank Hill tensor $[14,15]$ that depends on the specific ellipsoidal shape of domain $\Omega$. Inverting (5) yields the effective compliance:

$$
\left(\boldsymbol{S}^{\text {eff }}-\boldsymbol{S}^{0}\right)^{-1}=\left[\left(1 / V_{\Omega}\right) \sum_{i} V_{i} \boldsymbol{H}^{(i)}\right]^{-1}-\boldsymbol{Q}_{\Omega}
$$

In this form, Maxwell's covers mixtures of inhomogeneities of diverse shapes. Note that the noninteraction approximation is recovered if the first term in 
the brackets is much larger than the second one; in other words, tensor $\boldsymbol{Q}_{\Omega}$ is the quantity that describes the difference between Maxwell's scheme and the NIA. Alternatively, the Maxwell scheme can be formulated in terms of stiffness contribution tensors $N$.

We note certain logical inconsistency of the scheme: in ascribing to homogenized domain $\Omega$ the effective properties, interactions between inhomogeneities within $\Omega$ are assumed to be taken into account (otherwise, the scheme would have coincided with the NIA), whereas in considering the far fields generated by inhomogeneities, the interactions are disregarded. In other words, the lefthand part of the equation (4) is sensitive to the shape of $\Omega$ whereas the right-hand part is not. This is not fully consistent: the interaction effect experienced by an inhomogeneity can be characterized by placing it into the field $\sigma^{\infty}+\Delta \sigma$ where $\Delta \sigma$ is the correction due to interaction with neighbors within $\Omega$, and the corresponding correction to the far field generated by this inhomogeneity is of the first order in $\Delta \sigma$. This discrepancy leads to sensitivity of the predictions to the shape of $\Omega$ (indeed, as shown in $[16,17]$, incorporating pair interactions into the Maxwell scheme eliminates the sensitivity of the prediction to the shape of $\Omega$ ). The question arises on the optimal (from the viewpoint of accuracy of the scheme) choice of the shape. In the isotropic cases (fully random orientations, or spherical inhomogeneities), the spherical shape of $\Omega$ appears to be appropriate since there are no preferred directions. This choice may, possibly, be reasonable in cases of overall anisotropy as well (this assumes ascribing anisotropic properties, of the same symmetry as the effective ones, to the spherical domain $\Omega$ ), provided the type of the overall anisotropy can be specified a priori.

\section{The choice of shape of the effective inhomogeneity}

As discussed above, the shape of the effective inhomogeneity is one of the key parameters in Maxwell homogenization scheme. This shape has to obey the following two requirements:

- The shape of $\Omega$ should be ellipsoidal. Otherwise, one cannot proceed from formula (4) to (5) and thus cannot separate the two effects, of the domain shape and of the elastic contrast;

- The shape of $\Omega$ should properly reflect the shapes of individual inhomogeneities, their orientations and properties as discussed in the text to follow.

We now specify the requirement $B$ using certain known results: the one for isotropic composites [8, 18] and the one for a composite with parallel ellipsoidal inhomogeneities [9]. We utilize these results in conjunction with our observations on connections between the tensors entering expression (6), to come up with general recommendation regarding the shape of $\Omega$ (of the effective inhomogeneity in Maxwell scheme), for an arbitrary composite material (that may be anisotropic and may contain several sets of inhomogeneities).

\subsection{Isotropic distribution of identical inhomogeneities}

Since the only isotropic ellipsoidal shape is a sphere, the effective inhomogeneity has to be spherical so that

$$
\boldsymbol{Q}_{\Omega}=3 K_{0} \alpha_{K}\left(\frac{1}{3} \boldsymbol{I I}\right)+2 \mu_{0} \alpha_{G}\left(\boldsymbol{J}-\frac{1}{3} \boldsymbol{I I}\right)
$$

where $J_{i j k l}=\left(\delta_{i k} \delta_{l j}+\delta_{i l} \delta_{k j}\right) / 2, I I_{i j k l}=\delta_{i j} \delta_{k l}$ and

$$
\alpha_{K}=\frac{2}{3} \frac{1-2 v_{0}}{1-v_{0}} \text { and } \alpha_{\mu}=\frac{1}{15} \frac{7-5 v_{0}}{1-v_{0}}
$$

Note that the shape of individual inhomogeneities may be arbitrary, not necessarily ellipsoidal. For example, Giraud and Sevostianov [19] used Maxwell scheme to calculate the effective elastic properties of a material containing randomly oriented concave superspherical pores. In the case of randomly oriented spheroids $[11,20]$

$$
\begin{aligned}
& \frac{K_{e f f}}{K_{0}}=\frac{1-\alpha_{K} \sum_{i} \varphi_{i} B_{i}}{1+\left(1-\alpha_{K}\right) \sum_{i} \varphi_{i} B_{i}} ; \\
& \frac{G_{e f f}}{G_{0}}=\frac{1-\alpha_{G} \sum_{i} \varphi_{i} C_{i}}{1+\left(1-\alpha_{G}\right) \sum_{i} \varphi_{i} C_{i}}
\end{aligned}
$$

Formulas (9) coincide with the ones given in $[8,18,21]$.

\subsection{Parallel identical ellipsoidal inhomogeneities}

In this case,

$$
\frac{1}{V} \sum_{i} V_{i} \mathbf{H}^{i}=\varphi \mathbf{H}
$$

where $\varphi$ is the volume fraction of the inhomogeneities. McCartney [9] showed that the best choice of the shape of the effective inclusion is the shape of an individual inhomogeneity; thus $\boldsymbol{Q}_{\Omega}=\boldsymbol{Q}$, where $\boldsymbol{Q}$ is Hill's tensor of an individual inhomogeneity. Then the expression (6) for the effective compliance takes the form

$$
\begin{gathered}
\mathbf{S}^{\text {eff }}=\boldsymbol{S}^{0}+\varphi\left\{\left(\boldsymbol{S}^{1}-\boldsymbol{S}^{0}\right)^{-1}+(1-\varphi) \boldsymbol{Q}\right\}^{-1}= \\
=\mathbf{S}^{0}+\varphi\left\{(1-\varphi) \boldsymbol{H}^{-1}+\varphi\left(\boldsymbol{S}^{1}-\boldsymbol{S}^{0}\right)^{-1}\right\}^{-1}
\end{gathered}
$$

Thus, interactions in Maxwell's scheme are reflected only via tensor $\boldsymbol{Q}_{\Omega}$ that depends on the shape of the inhomogeneities and elastic constants of the matrix and inhomogeneities (in the case of multiphase materials it also depends on the partial volume fractions of the phases). 


\section{Maxwell scheme as one of effective field methods}

Maxwell's scheme can be considered as a version of the effective field method. Indeed:

$$
\begin{gathered}
\langle\boldsymbol{\varepsilon}\rangle=\mathbf{S}^{e f f}: \boldsymbol{\sigma}^{\infty}=\mathbf{S}^{0}: \boldsymbol{\sigma}^{\infty}+\left[\left(\sum_{i} \varphi_{i} \mathbf{H}^{(i)}\right)^{-1}-\mathbf{Q}^{\Omega}\right]^{-1}: \boldsymbol{\sigma}^{\infty}= \\
=\mathbf{S}^{0}: \boldsymbol{\sigma}^{\infty}+\sum_{i} \varphi_{i} \mathbf{H}^{(i)}: \underbrace{\left[\mathbf{J}-\mathbf{Q}^{\Omega}:\left(\sum_{i} \varphi_{i} \mathbf{H}^{(i)}\right)\right]^{-1}: \boldsymbol{\sigma}^{\infty}}_{\boldsymbol{\sigma}^{\text {eff }}}
\end{gathered}
$$

In the context of geophysical applications, Kuster and Toksöz [21] proposed a model for wave propagation in a media with randomly oriented spheroidal inhomogeneities that - as far as the effective elastic properties are concerned - coincides with Maxwell's model, with the domain $\Omega$ taken to be spherical. In the case of spherical inhomogeneities, the Kuster-Toksöz' scheme also coincides with the Mori-Tanaka-Benveniste one [22].

\section{References}

1. J.C. Maxwell, A Treatise on Electricity and Magnetism (Clarendon Press, Oxford, 1873)

2. Lord Rayleigh, Philosophical Magazine, 34, 481-502 (1892)

3. I. Sevostianov and M. Kachanov, M. International Journal of Engineering Science, 58, 124-128 (2012)

4. D.J. Jeffrey, Proceedings of the Royal Society of London, A 335, 355-367 (1973)

5. K.Z. Markov, Heterogeneous Media: Micromechanics Modeling Methods and Simulations, (eds. K.Z. Markov and L. Preziozi) (Birkhauser, Boston, 1-162, 2000).

6. G.W. Milton, The Theory of Composites (Cambridge University Press, Cambridge, 2002)

7. C.P. Wong and R.S. Bollampally, Journal of Applied Polymer Science, 3396-3403, 74 (1999)

8. L.N. McCartney and A. Kelly, Proceedings of the Royal Society of London, A464, 423-446 (2008)

9. L.N. McCartney, Philosophical Magazine, 90, 4175 4207 (2010)

10. V. Levin, S. Kanaun, and M. Markov, International Journal of Engineering Science, 75-8661 (2012)

11. I. Sevostianov and A. Giraud, International Journal of Engineering Science, 64, 23-36 (2013)

12. I. Sevostianov and M. Kachanov, International Journal of Solids and Structures, 48, 2340-2348 (2011)

13. I. Sevostianov and M. Kachanov, Journal of the Mechanics and Physics of Solids, 50, 253-282 (2002)

14. R. Hill, Journal of the Mechanics and Physics of Solids, 11, 357-372 (1963)
15. T.T. Wu. International Journal of Solids and Structures, 2, 1-8 (1966)

16. V.I. Kushch, I. Sevostianov, and A.S. Belyaev, Mechanics of Materials, 89, 1-11 (2015)

17. V.I. Kushch and I. Sevostianov, International Journal of Engineering Science, 98, 36-50 (2016)

18. L. Shen and S. Yi, International Journal of Solids and Structures 38, 5789-5805 (2001)

19. A. Giraud and I. Sevostianov, International Journal of Rock Mechanics and Mining Sciences, 62, 23-27 (2013).

20. I. Sevostianov, Mechanics of Materials, 75 45-59 (2014)

21. G.T. Kuster and M.N. Toksöz, Geophysics, 39, $587-$ 606 (1974)

22. J. Berryman and P. Berge, Mechanics of Materials, 22 149-164 (1996) 